\title{
Arachidonate 12-lipoxygenase may serve as a potential marker and therapeutic target for prostate cancer stem cells
}

\author{
BO YIN ${ }^{1}$, YANG YANG ${ }^{2}$, ZHIQIANG ZHAO ${ }^{2}$, YU ZENG $^{3}$, STEVEN M. MOONEY $^{3}$, \\ MING LI ${ }^{1}, X_{U E W E N ~ X U^{1}}$, YONGSHENG SONG ${ }^{1}$, BIN WU $^{1}$ and ZHIBO YANG ${ }^{4}$ \\ ${ }^{1}$ Department of Urology, Shengjing Hospital of China Medical University, 36 Sanhao Street, Shenyang 110004; \\ ${ }^{2}$ Department of Urology, Mudanjiang Medical College, 3 Tongxiang Street, Mudanjiang 157011, P.R. China; \\ ${ }^{3}$ Department of Urology, James Buchanan Brady Urological Institute, The Johns Hopkins University School of \\ Medicine, 600 North Wolfe Street, Baltimore, MD 21287; ${ }^{4}$ Department of Genitourinary Medical Oncology, \\ The University of Texas M.D. Anderson Cancer Center, 1515 Holcombe Blvd., Houston, TX 77030, USA
}

Received November 1, 2010; Accepted December 21, 2010

DOI: $10.3892 /$ ijo.2011.901

\begin{abstract}
The presence of arachidonate 12-lipoxygenase (12-LOX) potentiates prostate cancer $(\mathrm{PCa})$ progression and therefore may be a good therapeutic target and/or a potential diagnostic predictor for $\mathrm{PCa}$. In this study, we examined the expression of 12-LOX in PCa stem cells (PCa SCs) to test if it can serve as a unique marker and therapeutic target for $\mathrm{PCa}$ SCs. To this end, we isolated the cancer stem cell-like side population (SP) cells from the human PCa cell line DU-145 by a flow cytometry-based SP technique. The isolated DU-145 SP cells comprised a small population of the DU-145 cells. The SP cells had an up-regulation of ATP-binding cassette sub-family G member 2 (ABCG2) which enables these cells to efflux vital dyes and chemotherapeutic drugs. Furthermore, we detected a strong up-regulation of 12-LOX in these DU-145 SP cells compared to the parental DU-145 cells by RT-PCR and Western blot approaches. We also detected 12-LOX overexpression in PCa SCs in human PCa tissue samples by paraffin-section based immunofluorescent 4-channel confocal microscopy. However, no 12-LOX was detected in normal prostate epithelial SCs in normal prostate tissue samples. These multiple lines of evidence support the possibility that 12-LOX may serve as a unique marker and therapeutic target for PCa SCs.
\end{abstract}

\section{Introduction}

Prostate cancer $(\mathrm{PCa})$ is the most frequently diagnosed cancer and a leading cause of cancer death in American males (1).

Correspondence to: Dr Bo Yin, Department of Urology, Shengjing Hospital of China Medical University, 36 Sanhao Street, Shenyang 110004, P.R. China

E-mail: yinbowzf@163.com

Key words: arachidonate 12-lipoxygenase, prostate cancer, stem cells, cancer stem cells, side population
PCa is considered particularly heterogeneous since it is generally composed of cells with different phenotypic characteristics, as well as different proliferative and malignant potentials. Accumulating evidence suggests that only a rare, phenotypically distinct subset of cells known as cancer stem cells (CSCs) have the capacity to form new tumors and are responsible for fueling a tumor's growth (2). The importance of CSCs in tumorinitiation has been firmly established in leukemia (3) and recently reported for a variety of solid tumors (4-6), suggesting that only a small fraction of cells in the tumor, namely the CSCs drive the formation and progression of tumors. Based on high surface expression of the markers, CD44+/integrin $\alpha_{2} \beta_{1}{ }^{\text {high }} / \mathrm{CD} 133+$, Collins et al (7) have pioneered the identification and isolation of CSCs from primary and metastatic $\mathrm{PCa}$. Very recently, some exciting data have been generated using these prostate cancer stem cell ( $\mathrm{PCa} \mathrm{SCs}$ ) markers by various groups $(8,9)$.

The hypothesis that CSCs are the origin of cancer, has profound implications for cancer therapy since the immediate goal should be the identification and selective killing of CSCs in an attempt to eradicate cancer cells. In other words, current cancer therapies should target CSCs rather than the cancer cells that are generated by them. However, emerging evidence has revealed that normal stem cells (SCs) and CSCs share similar properties. For example, $\mathrm{PCa} \mathrm{SCs}$ have been identified using the normal prostate epithelial SCs markers CD44+/ integrin $\alpha_{2} \beta_{1}{ }^{\text {high }} / \mathrm{CD} 133+(7)$. Because normal prostate SCs also carry CD133 (10) and some other markers on their surfaces, they could be destroyed by drugs that target PCa SCs by using these markers. Currently seeking additional marker(s) unique to CSCs is a high priority in enabling safer targeting.

Recent studies have implicated arachidonate 12-lipoxygenase (12-LOX), an enzyme metabolizing arachidonic acid to form 12(S)-hydroxyeicosatetraenoic acid (HETE), as a biological determinant for PCa progression (11). However, the characterization such as expression and function of 12-LOX in CSCs especially in PCa SCs has not been documented.

The present study was undertaken to test whether 12-LOX may serve as a unique marker to PCa SCs. We applied flow 
cytometry-based side population (SP) analysis to isolate cancer stem cell-like SP cells to define the expression of 12-LOX. We also tested the expression of 12-LOX in PCa SCs and normal prostate epithelial SCs identified in tissue samples by paraffin-section based immunofluorescent 4-channel laser scanning confocal microscopy.

\section{Materials and methods}

Cell line and tissue samples. The prostate cancer cell line DU-145 was purchased from American Type Culture Collection (Rockville, MD, USA). The cells were routinely cultured in RPMI-1640 (Invitrogen, CA, USA) supplemented with $10 \%$ fetal bovine serum (FBS) at $37^{\circ} \mathrm{C}$ in incubator with humidified air and 5\% carbon dioxide.

Seven paraffin-embedded prostate adenocarcinoma samples and ten paraffin-embedded normal prostate tissue samples were obtained from the Department of Pathology of the University of Texas M.D. Anderson Cancer Center. The 7 cases of prostate adenocarcinoma samples were collected from the patients undergoing radical prostatectomy (age range 54-69 years). The histological and pathological stage of tumors was established in accordance with the tumor-nodemetastasis system recommended by the American Joint Committee on Cancer which showed stage T2 in 4 cases, T3a in 2 cases and T3b in 1 case. Of these prostate adenocarcinoma samples, 4 were Gleason score 5-6, 2 were Gleason score 7 and 1 was Gleason score 9 . The 10 cases of normal prostate tissue samples were collected from the patients undergoing radical cystectomy for transitional cell carcinoma of the bladder. Informed consent was obtained from each participant. This study protocol was approved by the institutional review board of the University of Texas M.D. Anderson Cancer Center.

Flow cytometry-based side population (SP) analysis. We optimized the protocol for isolating SP cells from human cancer cell lines as follows: cells were incubated with Hoechst 33342 (Molecular Probes, OR, USA) at a final concentration of $5 \mu \mathrm{g} / \mathrm{ml}$ for $45 \mathrm{~min}$ in the absence or presence of $150 \mu \mathrm{M}$ verapamil (Sigma-Aldrich, MO, USA) followed by staining with propidium iodine (PI) for the exclusion of dead cells. Cells were then subjected to fluorescence-activated cell sorting (FACS) analysis (M.D. Anderson Flow Cytometry and Imaging Core Lab) and the fluorescence was measured using two filters, $450 \mathrm{~nm}$ (Hoechst blue) and $670 \mathrm{~nm}$ (Hoechst red). SP cells were then cultured in DMEM/F12 medium (Invitrogen, CA, USA) supplemented with 2\% B27, $40 \mathrm{ng} / \mathrm{ml}$ basic fibroblast growth factor (bFGF; Sigma-Aldrich, MO, USA) and $20 \mathrm{ng} / \mathrm{ml}$ endothelial growth factor (EGF; SigmaAldrich, MO, USA).

Reverse transcriptase-polymerase chain reaction (RT-PCR). Total RNA was isolated from homogenized cells using RNeasy Mini kit (Qiagen, Austin, TX, USA) following the manufacturer's instructions with an additional step of treatment with DNase I (Invitrogen, CA, USA) to remove any genomic DNA contamination. RNA samples were then stored at $-80^{\circ} \mathrm{C}$ until further use. cDNA was synthesized using the iScript cDNA synthesis kit (Bio-Rad, CA, USA). The housekeeping gene GAPDH was amplified using synthesized cDNA to confirm integrity.

Primers for target genes were designed based on GeneBank sequences. 12-LOX: F-5'-CTCTGCCATTTCCTCACCAT-3' and R-5'-GCCATCGTCACATCTTCCTT-3', 152 bp; ATP-binding cassette sub-family $\mathrm{G}$ member 2 (ABCG2): F-5'-CCTGAGATCCTGAGCCTTTG-3' and R-5'-AAGCCAT TGGTGTTTCCTTG-3', 124 bp; and GAPDH: F-5'-GAGTCA ACGGATTTGGTCGT-3' and R-5'-GACAAGCTTCCCGTT CTCAG-3', 185 bp. PCR was carried out under the following conditions: for $12-\mathrm{LOX}$, denaturation for $30 \mathrm{sec}$ at $95^{\circ} \mathrm{C}$ followed by annealing for $30 \mathrm{sec}$ at $61^{\circ} \mathrm{C}$, then elongation for $30 \mathrm{sec}$ at $70^{\circ} \mathrm{C}, 35$ cycles total; ABCG 2 was identical to 12-LOX except that $63^{\circ} \mathrm{C}$ was used as annealing temperature; GAPDH was identical to ABCG2 except that only 30 cycles were used. Electrophoresis was done by loading $10 \mu \mathrm{l}$ of each sample on a $2 \%$ agarose gel, and visualized by ethidium bromide staining using the Bio-Imaging System (Ultra-Violet Products, Cambridge, UK). Positive expression was defined as those bands that electrophoresed with the expected size.

Western blot analysis. Twenty-five micrograms of protein were separated on a 10-20\% SDS-PAGE and transferred onto PVDF membrane (Millipore, MA, USA). The membrane was incubated with primary antibody overnight at $4{ }^{\circ} \mathrm{C}$ followed by incubation with horseradish peroxidase-conjugated secondary antibody, and developed with the Super Signal West Dura Extended Duration Substrate kit (Pierce, IL, USA). The primary antibody to 12-LOX (rabbit polyclonal antibody; Novus, CO, USA) was incubated at 1:500 overnight then 1:20,000 secondary antibody (goat anti-rabbit antibody; Santa Cruz, USA) was incubated for $2 \mathrm{~h}$ at room temperature. Actin was used as a loading control.

Immunofluorescent 4-channel laser scanning confocal microscopy. Paraffin-section based immunofluorescent 4-channel laser scanning confocal microscopy was applied to detect the expression of 12-LOX in PCa SCs and normal prostate SCs identified in tissue samples. Generally, after deparaffinization and antigen retrieval, slides were incubated with a cocktail of primary antibodies including mouse antiintegrin $\alpha_{2} \beta_{1}$ monoclonal antibody (Millipore, MA, USA), rat anti-CD133 monoclonal antibody (Novus, CO, USA) and rabbit anti-12-LOX polyclonal antibody (Novus) overnight at $4^{\circ} \mathrm{C}$. A cocktail of secondary antibodies including Alexa Fluor-350 labeled goat anti-mouse, Alexa Fluor-647 labeled goat anti-rat and Alexa Fluor-546 labeled goat anti-rabbit antibodies (Molecular Probes, Eugene, OR, USA) were applied and incubated for $45 \mathrm{~min}$ at room temperature. Slides were then incubated with the fourth primary antibody, fluorescein isothiocyanate (FITC)-conjugated rat anti-CD44 monoclonal antibody (Novus, CO, USA) for $30 \mathrm{~min}$ at room temperature and mounted in anti-fade solution (Molecular Probes). Digital micrographs were acquired using serial laser excitation on an Leica DM 6000B laser scanning confocal microscope, and then were imported into a Dell Power Edge 2200 computer for processing using the Lasersharp 3.0 program (Bio-Rad, CA, USA). In order to reduce the background noise, signal thresholds were set up according to the corresponding control slides. Selected images were representative of at least 

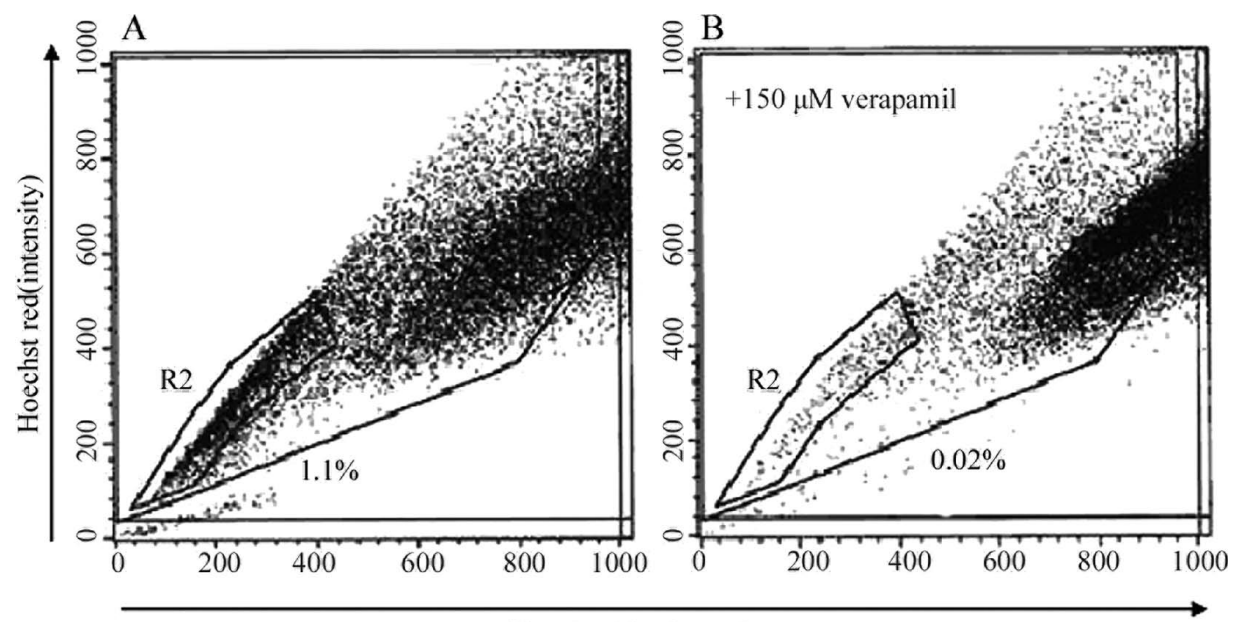

Hoechst blue(intensity)

Figure 1. Isolation of SP cells from the DU-145 cell line by Hoechst 33342 SP analysis in the absence (A) or presence (B) of verapamil.
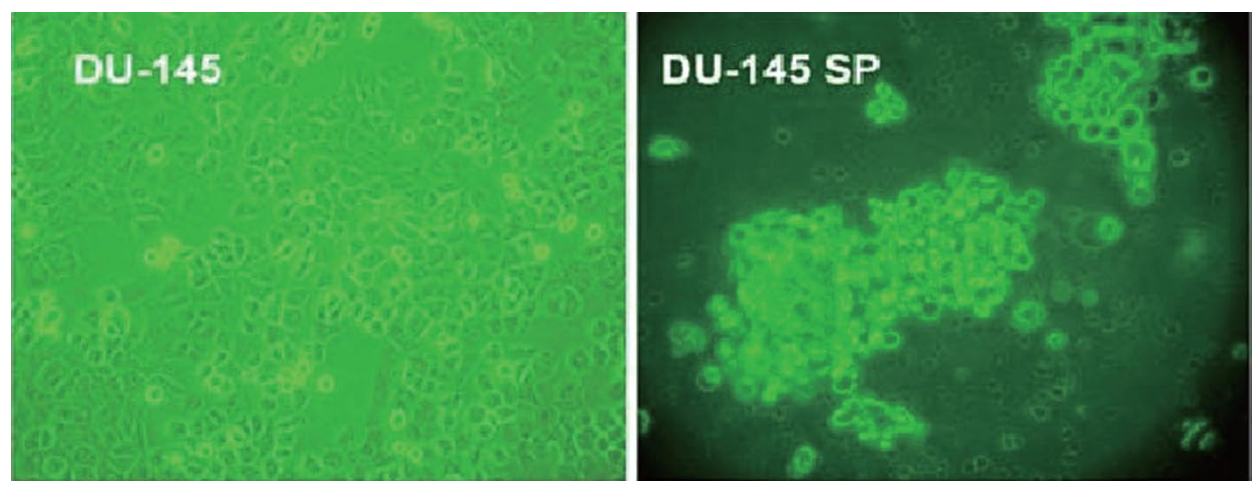

Figure 2. Morphologic features of DU-145 SP cells, as illustrated by phase contrast microscopy (x20).

10 microscopic fields analyzed for each condition. All the secondary antibodies were purchased from Molecular Probes (Eugene, OR, USA) and were excited at a wavelength of 647, 546 and 350, respectively. The FITC-conjugated rat anti-CD44 primary antibody was excited at a wavelength of 488 . All confocal images were acquired with a frame size of $512 x$ 512 pixels and were averaged four times. Sequential scanning was applied to avoid bleed-through. Slides without primary/ secondary antibody were used as negative controls.

\section{Results}

Identification of cancer stem cell-like SP cells from established human PCa cell line DU-145. To determine whether the human PCa cell line DU-145 contained SP cells, we removed the cells from the culture dish with trypsin and EDTA and stained them with fluorescent dye Hoechst 33342, which has been shown to be actively extruded from SP cells in various tissues by means of verapamil-sensitive ATP-binding cassette (ABC) transporters. We then analyzed the cells by flow cytometry-based SP technique. As shown in Fig. 1A, the DU-145 cell line contained a small population (about 1.1\%) of SP cells in the left lower quadrant of the FACS profile in the absence of verapamil. The SP population decreased dramatically in the presence of vera- pamil (Fig. 1B), indicating that the population was made of bona fide SP cells.

The isolated SP cells were cultured in the medium (see Materials and methods) for 3 weeks and then photographed while alive by a phase contrast microscope. The morphology of SP cells was different from parental DU-145 cells in that SP cells had a round body even though they were still attached to the dish (Fig. 2).

Since the efflux of Hoechst 33342 from SP cells is mainly through $\mathrm{ABC}$ transporters, we further examined the expression of $\mathrm{ABCG} 2$, which is one of the best characterized ABC transporters, between SP and non-SP cells. As compared to parental DU-145 cells, ABCG2 was found to be up-regulated in SP cells by RT-PCR (Fig. 3A).

Expression of 12-LOX in cancer stem cell-like SP cells isolated from human PCa cell line DU-145. After succeeding in isolating DU-145 SP cells, we further examined the expression of 12-LOX in cancer stem cell-like SP cells to determine if 12-LOX is a unique marker to PCa SCs. Firstly, we determined the mRNA expression of 12-LOX by RT-PCR. We detected an up-regulation of 12-LOX mRNA in SP cells as compared to parental DU-145 cells (Fig. 3B). Then we determined the protein expression of 12-LOX by Western blotting. We also 

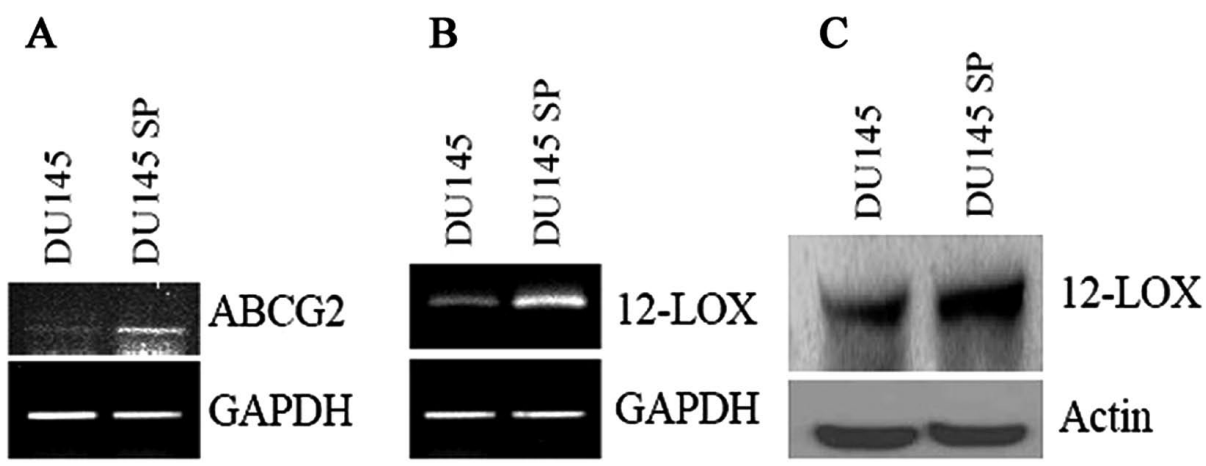

Figure 3. Expression of ABCG2 (A) by RT-PCR, up-regulation of 12-LOX by RT-PCR (B) and by Western blotting (C) in DU-145 SP cells as compared to parental DU-145 cells.
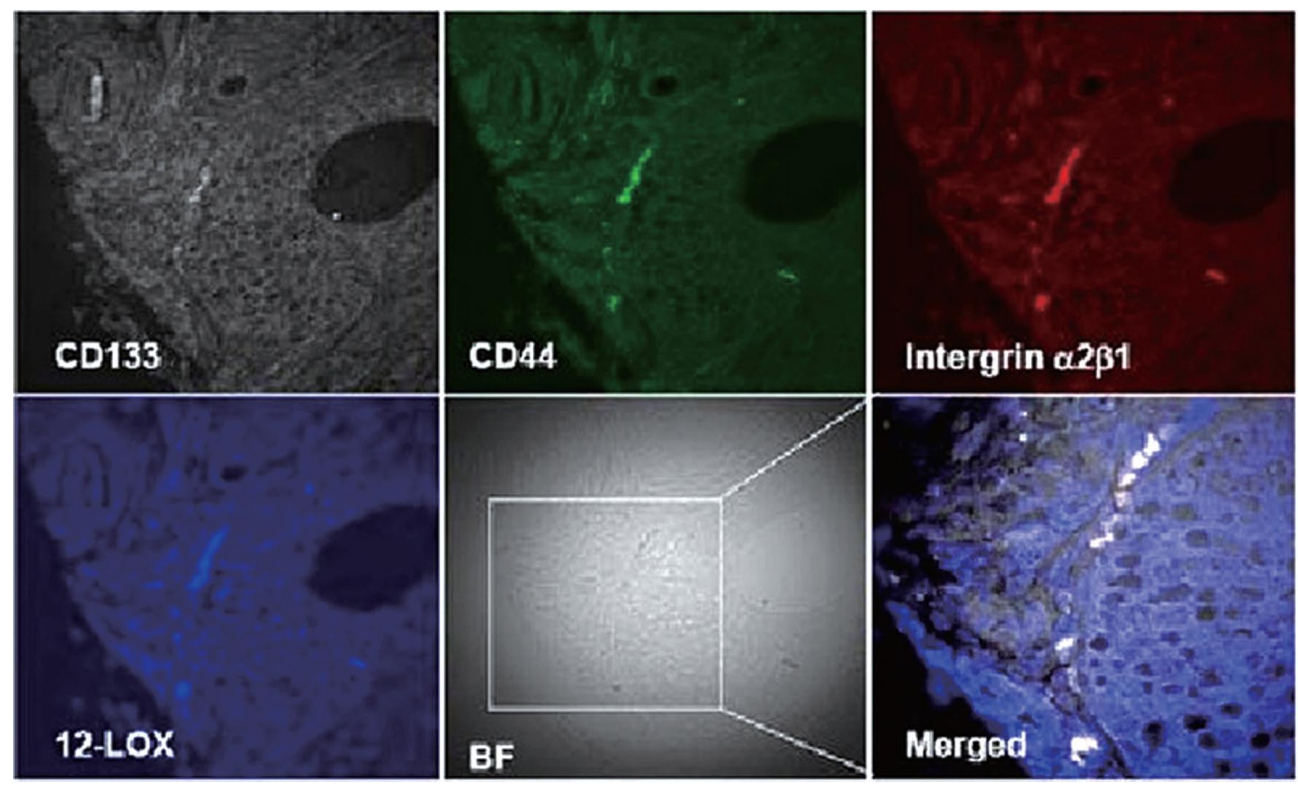

Figure 4. PCa SCs were identified by the markers of CD44+/integrin $\alpha_{2} \beta_{1}^{\text {high }} / \mathrm{CD} 133+$ in PCa tissue samples and overexpression of 12-LOX (blue) was shown by using 4-channel confocal microscopy (40x10). BF, bright field.

detected an up-regulated protein expression in SP cells (Fig. 3C), which is consistent with mRNA up-regulation. To test the possible correlation of PCa SCs marker to the SP phenotype, we further determined the coexpression of 12-LOX and CD133, a PCa SCs marker, in those SP cells by confocal microscopy. We detected an up-regulated coexpression of 12-LOX and CD133 (data not shown).

Expression of 12-LOX in PCa SCs and normal prostatic epithelial SCs identified in tissue samples. Immunofluorescent 4-channel laser scanning confocal microscopy was applied to detect the expression of 12-LOX in PCa SCs and normal prostatic epithelial SCs identified in tissue samples to further confirm that 12-LOX may serve as a unique marker to $\mathrm{PCa}$ SCs. Immunofluorescent 4-channel confocal microscopy enabled us to identify the PCa SCs or normal prostatic epithelial SCs by detecting the following markers: CD44+/integrin $\alpha_{2} \beta_{1}{ }^{\text {high }} / \mathrm{CD} 133+(7)$, and its fourth channel was used to study the expression of 12-LOX. Using this technique, we detected that 12-LOX was overexpressed in PCa SCs, which were identified by three markers of CD44+/integrin $\alpha_{2} \beta_{1}{ }_{1}^{\text {high }} / \mathrm{CD} 133+$ and located near basal membrane, in 7 cases of human prostate adenocarcinoma samples (Fig. 4) regardless of tumor grade and stage. Among them, 4 cases were strongly stained and 3 cases were weakly stained. On the contrary, 12-LOX was undetectable in those normal prostate epithelial SCs identified with those same three markers of CD44+/integrin $\alpha_{2} \beta_{1}{ }^{\text {high }} / \mathrm{CD} 133+$ in 10 normal prostate tissue samples (Fig. 5).

\section{Discussion}

According to the American Cancer Society, 186,320 new cases of PCa were diagnosed in the US in 2007. With an estimated 28,660 deaths in $2008, \mathrm{PCa}$ is a leading cause of cancer death in men (1). To improve the treatment outcome of $\mathrm{PCa}$, the mechanism of regulation of PCa progression needs to be investigated, promising novel targets for therapy need to be unveiled and promising novel therapies urgently need to be developed. 


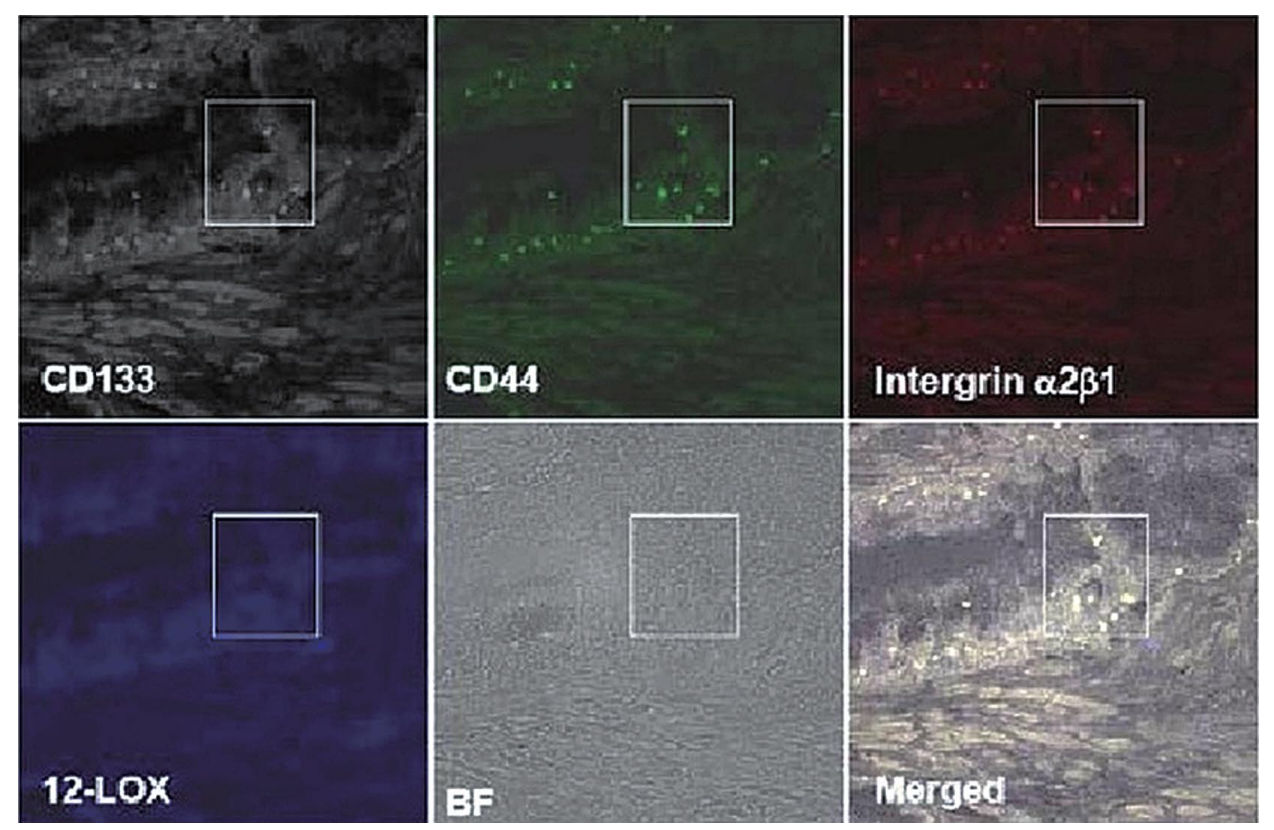

Figure 5. Normal prostatic epithelial SCs were identified by the markers of CD44+/integrin $\alpha_{2} \beta_{1}$ high/CD133+ in normal prostate tissue samples by using 4-channel confocal microscopy (40x10). 12-LOX (blue) was not detected. BF, bright field.

In recent years, SCs have become the focus of a tremendous amount of biomedical research because of their apparent potential for regenerative medicine $(12,13)$. There is now increasing evidence that tumor progression is driven by CSCs which are very malignant and possess a unique ability to initiate tumor progression (14). Thus, for cancer therapy to be curative, it probably must eliminate these CSCs, which is why it is important to identify and study CSCs.

Based on high surface expression of CD44+/integrin

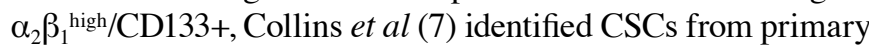
and metastatic $\mathrm{PCa}$. However, emerging evidence has revealed that normal prostatic epithelial SCs and PCa SCs share similar properties. Since normal prostate epithelial SCs carry CD133 and other markers, such as ABCG2, on their surfaces, they could inadvertently be destroyed by drugs targeting PCa SCs by using these markers. As a result, it is highly necessary to find additional marker(s) that are unique to CSCs, thus enabling more specific and safer targeting.

On the other hand, some studies have shown that certain metabolites of arachidonate, mainly the 12-LOX product 12(S)-HETE, play significant roles in the angiogenic and metastatic potentials of tumors. There are several lines of evidence implicating the involvement of 12 -LOX in $\mathrm{PCa}$ progression. First, 12-LOX expression was detected in human prostatic tumors and correlated to the clinical stage of disease (15). Second, overexpression of 12-LOX in human PCa cells stimulates angiogenesis and tumor growth (16). Third, an inhibitor of 12-LOX has been found effective against metastatic prostate tumor growth by inducing the release of cytochrome $\mathrm{C}$ from mitochondia which activates caspase 9, 7, 3 and eventually leads to apoptosis (17). Thus, 12-LOX is a potential predictor for the aggressiveness of $\mathrm{PCa}$ and may become a novel target for the development of anti-invasive and anti-metastatic agents in the treatment of PCa (18). However, the expression and function of 12-LOX in PCa SCs has not been documented and until now, a link between 12-LOX and PCa SCs in PCa progression has not been reported.

Two general approaches have been used to identify and characterize CSCs. First, SP cells isolated from cancers by flow cytometry-based SP technique have proven to be an attractive alternative to study CSCs (19). Second, molecular markers have been proposed to identify CSCs, e.g. CD44+/CD24- for breast cancer stem cells (20), CD34+/CD38- for leukemia stem cells (21) and CD44+/integrin $\alpha_{2} \beta_{1}{ }_{\text {high }} / \mathrm{CD} 133+$ for PCa SCs (7). The present study was undertaken by combining these two approaches to address whether 12-LOX may serve as a unique marker for PCa SCs.

Flow cytometry-based SP technique relies on the ability of SP cells to efflux Hoechst dye 33342 (22). These SP cells are identified according to their ability to efflux Hoechst dye 33342 at a greater rate than other cells within a specific tissue. The degree of efflux activity correlates with the maturation state, such that cells exhibiting the highest efflux activity are the most primitive or the least restricted in terms of differentiation potential (23). It has been widely accepted that PCa SP cells likely contain primitive CSCs (24-26).

It is believed that the overexpression of membrane transporters is responsible for the dye efflux mechanism in SP cells. The high drug efflux capacity of these SP cells correlates with the strong expression of drug-transporter proteins including ABCG2, as would be expected given the ability to efflux vital dyes is the basis upon which the cells are sorted from these tumors. This SP approach has been used widely to enrich SCs. For example, LAPC-9 (a PCa cell line from bone metastasis) cells have $\sim 0.1 \%$ SP cells, which is about 1,000 times more tumorigenic than the non-SP cells and the SP-derived tumors can be xenotransplanted for multiple generations, strongly suggesting that the PCa SP cells likely contain primitive CSCs. Despite the fact that there are undoubtedly technical variations 
in the isolation, it is still believed in general that the SP sorting is a good place to start the search for resident SCs in a particular organ especially when the phenotype of the cells in question is not known. Considering the purity of the SP population being isolated, researchers started to refer to this group of cells as 'cancer stem cell-like SP cells'.

In the present study, we succeeded in isolating SP cells from the PCa cell line DU-145 by flow cytometry-based SP technique. We found that DU-145 SP cells were only about $1.1 \%$ of total cells. The SP population decreased dramatically in the presence of verapamil and SP cells have an up-regulation of ABCG2, indicating that this population was made up of bona fide SP cells. Our study outlined a successful strategy for isolating cancer stem cell-like SP cells, which made it possible to further study $\mathrm{PCa}$ SCs, especially in regard to the function of 12-LOX in PCa SCs. Then we determined the expression of 12-LOX in DU-145 SP cells by RT-PCR and Western blotting. Our data showed that as compared to parental DU-145 cells, 12-LOX was up-regulated in SP cells at mRNA and protein levels, indicating that 12 -LOX may serve as a unique marker for PCa SCs. Furthermore, we examined the expression of 12-LOX in PCa SCs and normal prostatic epithelial SCs identified in tissue samples. Immunofluorescent 4-channel confocal microscopy enabled us to identify the PCa SCs or normal prostatic epithelial SCs by detecting the following markers: CD44+/integrin $\alpha_{2} \beta_{1}{ }^{\text {high} / C D 133+, ~ a n d ~ i t s ~ f o u r t h ~ c h a n n e l ~ w a s ~}$ used to study the expression of 12-LOX. We detected that 12-LOX was overexpressed in PCa SCs regardless of tumor grade and stage, but 12-LOX was undetectable in those normal prostate epithelial SCs. Although a larger number of tissue samples are needed to confirm the expression of 12-LOX in PCa SCs, our preliminary data did indicate that $12-\mathrm{LOX}$ has a potential to serve as a unique marker and therapeutic target for PCa SCs.

\section{Acknowledgements}

This study was partially sponsored by Liaoning Provincial Education Office Fund in China (Grant no. 2009A735). We thank Zhihong Zong for excellent technical assistance.

\section{References}

1. Heppner GH: Tumor heterogeneity. Cancer Res 44: 2259-2265, 1984.

2. Hamburger AW and Salmon SE: Primary bioassay of human tumor stem cells. Science 197: 461-463, 1977.

3. Liu J and Jiang G: CD44 and hematologic malignancies. Cell Mol Immunol 3: 359-365, 2006.

4. Sheridan C, Kishimoto H, Fuchs RK, et al: CD44+/CD24- breast cancer cells exhibit enhanced invasive properties: an early step necessary for metastasis. Breast Cancer Res 8: R59, 2006.

5. Wang HS, Hung Y, Su CH, et al: CD44 cross-linking induces integrin-mediated adhesion and transendothelial migration in breast cancer cell line by up-regulation of LFA-1 (alpha L beta2) and VLA-4 (alpha4beta1). Exp Cell Res 304: 116-126, 2005.
6. Bhatt RI, Brown MD, Hart CA, Gilmore P, Ramani VA, George NJ and Clarke NW: Novel method for the isolation and characterisation of the putative prostatic stem cell. Cytometry A 54: 89-99, 2003.

7. Collins AT, Berry PA, Hyde C, Stower MJ and Maitland NJ: Prospective identification of tumorigenic prostate cancer stem cells. Cancer Res 65: 10946-10951, 2005.

8. Li H, Zhou J, Miki J, et al: Telomerase-immortalized non-malignant human prostate epithelial cells retain the properties of multipotent stem cells. Exp Cell Res 314: 92-102, 2008.

9. Wei C, Guomin W, Yujun L and Ruizhe Q: Cancer stem-like cells in human prostate carcinoma cells DU145: the seeds of the cell line? Cancer Biol Ther 6: 763-768, 2007.

10. Richardson GD, Robson CN, Lang SH, Neal DE, Maitland NJ and Collins AT: CD133, a novel marker for human prostatic epithelial stem cells. J Cell Sci 117: 3539-3545, 2004.

11. Pirtskhalaishvili G, Hrebinko RL and Nelson JB: The treatment of prostate cancer: an overview of current options. Cancer Pract 9: 295-306, 2001

12. Fuchs E and Segre JA: Stem cells: a new lease on life. Cell 100: $143-155,2000$.

13. Haraguchi N, Utsunomiya T, Inoue H, Tanaka F, Mimori K, Barnard GF and Mori M: Characterization of a side population of cancer cells from human gastrointestinal system. Stem Cells 24: 506-513, 2006.

14. Kim CF, Jackson EL, Woolfenden AE, et al: Identification of bronchioalveolar stem cells in normal lung and lung cancer. Cell 121: 823-835, 2005.

15. Pidgeon GP, Tang K, Cai YL, Piasentin E and Honn KV: Overexpression of platelet-type 12-lipoxygenase promotes tumor cell survival by enhancing alpha(v)beta(3) and alpha(v)beta(5) integrin expression. Cancer Res 63: 4258-4267, 2003.

16. Nie D, Krishnamoorthy S, Jin R, et al: Mechanisms regulating tumor angiogenesis by 12-lipoxygenase in prostate cancer cells. J Biol Chem 281: 18601-18609, 2006.

17. Tong WG, Ding XZ, Witt RC and Adrian TE: Lipoxygenase inhibitors attenuate growth of human pancreatic cancer xenografts and induce apoptosis through the mitochondrial pathway. Mol Cancer Ther 1: 929-935, 2005.

18. Tang K, Finley RL Jr, Nie D and Honn KV: Identification of 12-lipoxygenase interaction with cellular proteins by yeast two-hybrid screening. Biochemistry 39: 3185-3191, 2000.

19. Hadnagy A, Gaboury L, Beaulieu R and Balicki D: SP analysis may be used to identify cancer stem cell populations. Exp Cell Res 312: 3701-3710, 2006.

20. Ponti D, Costa A, Zaffaroni N, et al: Isolation and in vitro propagation of tumorigenic breast cancer cells with stem/ progenitor cell properties. Cancer Res 65: 5506-5511, 2005.

21. Raaijmakers MH, de Grouw EP, Heuver LH, et al: Breast cancer resistance protein in drug resistance of primitive CD34+38- cells in acute myeloid leukemia. Clin Cancer Res 11: 2436-2444, 2005.

22. Hirschmann-Jax C, Foster AE, Wulf GG, et al: A distinct 'side population' of cells with high drug efflux capacity in human tumor cells. Proc Natl Acad Sci USA 101: 14228-14233, 2004.

23. Challen GA and Little MH: A side order of stem cells: the SP phenotype. Stem Cells 24: 3-12, 2006.

24. Patrawala L, Calhoun T, Schneider-Broussard R, Zhou J, Claypool K and Tang DG: Side population is enriched in tumorigenic, stem-like cancer cells, whereas ABCG2+ and ABCG2-cancer cells are similarly tumorigenic. Cancer Res 65: 6207-6219, 2005.

25. Gong Z, Hebert JR, Bostick RM, et al: Common polymorphisms in 5-lipoxygenase and 12-lipoxygenase genes and the risk of incident, sporadic colorectal adenoma. Cancer 109: 849-857, 2007.

26. Rásó E, Döme B, Somlai B, Zacharek A, Hagmann W, Honn KV and Tímár J: Molecular identification, localization and function of platelet-type 12-lipoxygenase in human melanoma progression, under experimental and clinical conditions. Melanoma Res 14: $245-250,2004$ 